\title{
Editorial Statistics and Best Reviewers Award for 2018
}

\author{
Toyoaki Murohara, MD, $\mathrm{PhD}$
}

Dear Colleagues,

On behalf of the Circulation Journal, I am pleased to announce the editorial statistics for 2018, and the recipients of the Best Reviewers Awards for 2018.

\section{Editorial statistics for 2018}

As shown in Figure 1, we received 1,395 manuscripts in 2018, for publication in the Journal, including 1,046 original papers (924 clinical and 122 experimental), 16 invited review articles, 65 invited editorials, 47 rapid
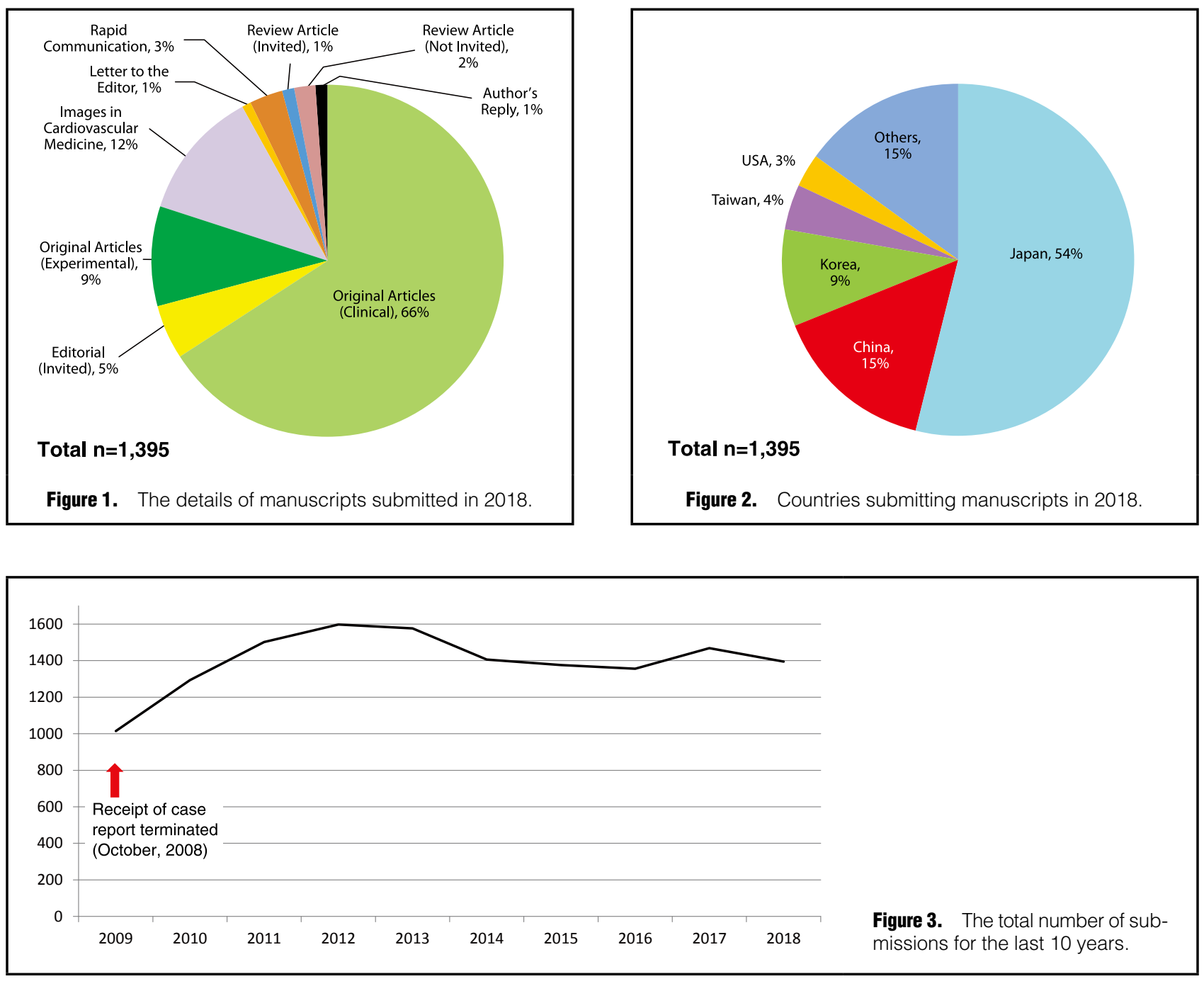

J-STAGE Advance Publication released online January 19, 2019

Department of Cardiology, Nagoya University Graduate School of Medicine, Nagoya, Japan

Mailing address: Toyoaki Murohara, MD, PhD, Department of Cardiology, Nagoya University Graduate School of Medicine, 65

Tsurumai, Showa-ku, Nagoya 466-8550, Japan. E-mail: murohara@med.nagoya-u.ac.jp

ISSN-1346-9843 All rights are reserved to the Japanese Circulation Society. For permissions, please e-mail: cj@j-circ.or.jp 


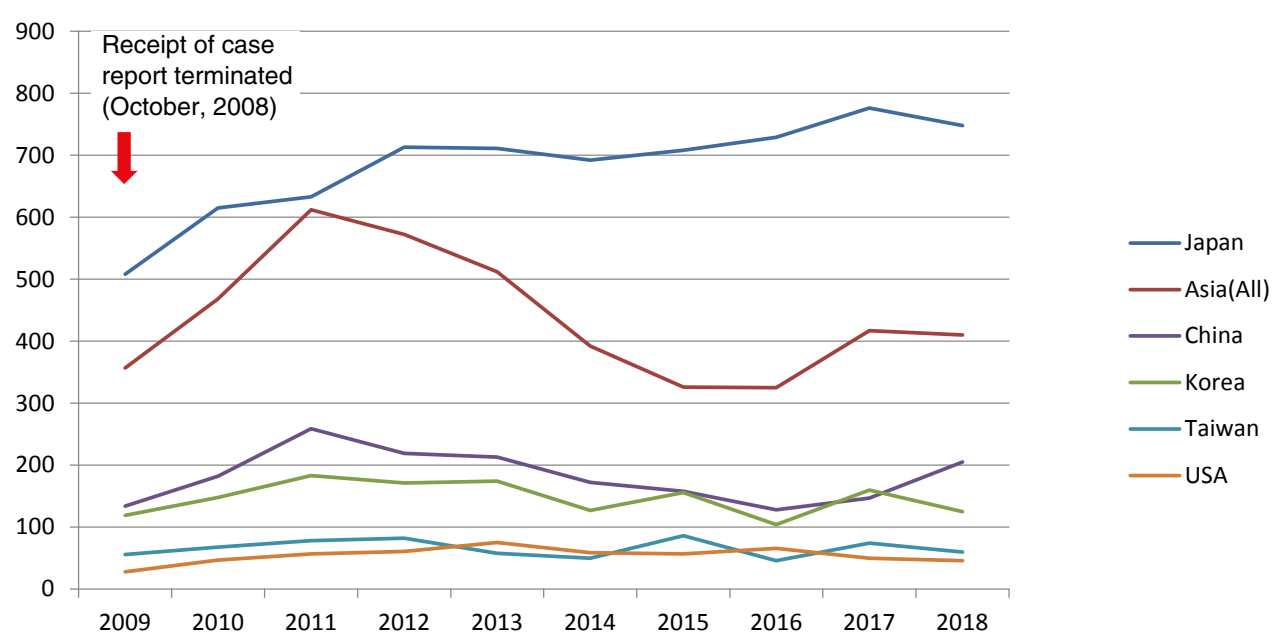

Figure 4. The number of submissions for the last 10 years in top 5 countries. Asia (All): all Asian countries except Japan.

communications, and 173 images in cardiovascular medicine. Approximately half were submitted from Japan, followed by China, Korea, Taiwan, and USA (Figure 2). For the last 10 years, the number of submissions has been steadily increasing even after we terminated publication of case reports in October 2008 (Figure 3). The abovementioned 5 countries have remained the top 5 countries in terms of paper submissions over that period (Figure 4). The acceptance rate for original manuscripts in 2018 was

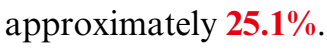

\section{Best Reviewers Awards for 2018}

In 2018, a total of 920 reviewers kindly reviewed the manuscripts submitted. In appreciation of their valuable contributions, we have selected the 20 Best Reviewers of the Year 2018 as follows:

\section{Hideki Ishii, MD}

(Nagoya University Graduate School of Medicine, Aichi)

Number of reviews: 21

RPS: 128.0

Previous awards: 2008, 2011, 2012, 2015, 2016, 2017

\section{Hiroshi Asanuma, MD}

(Meiji University of Integrative Medicine, Kyoto)

Number of reviews: 18

RPS: 115.0

Previous awards: 2009, 2010, 2011, 2012, 2013, 2014, 2015, 2016, 2017

\section{Eitaro Kodani, MD}

(Nippon Medical School Tama-Nagayama Hospital, Tokyo)

Number of reviews: 11

RPS: 82.0

Previous awards: 2017

\section{Akiomi Yoshihisa, MD}

(Fukushima Medical University, Fukushima)

Number of reviews: 13

RPS: 79.2

\section{Osamu Tsukamoto, MD}

(Osaka University Graduate School of Medicine, Osaka)

Number of reviews: 21

RPS: 123.0

Previous awards: 2012, 2013, 2014, 2015, 2016, 2017

\section{Shin Ito, MD}

(National Cerebral and Cardiovascular Center, Osaka) Number of reviews: 17

RPS: 112.0

Previous awards: 2017

\section{Naohiko Takahashi, MD}

(Oita University Faculty of Medicine, Oita)

Number of reviews: 15

RPS: 79.3

Previous awards: 2011, 2012, 2013, 2014, 2016, 2017

\section{Takahiro Okumura, MD}

(Nagoya University Graduate School of Medicine, Aichi)

Number of reviews: 13

RPS: 76.0 


\section{Shoji Sanada, MD}

(Osaka University Graduate School of Medicine,

Osaka)

Number of reviews: 13

RPS: 75.0

Previous awards: 2008, 2009, 2010, 2011, 2012, 2013,

2014, 2015, 2016, 2017

\section{Shinsuke Miyazaki, MD}

(University of Fukui Faculty of Medical Sciences,

Fukui)

Number of reviews: 12

RPS: 68.0

Previous awards: 2015, 2017

\section{Eiichi Watanabe, MD}

(Fujita Health University School of Medicine, Aichi)

Number of reviews: 11

RPS: 59.2

\section{Kentaro Hayashida, MD}

(Keio University School of Medicine, Tokyo)

Number of reviews: 16

RPS: 58.4

\section{Yasushi Mukai, MD}

(Kyushu University, Fukuoka)

Number of reviews: 9

RPS: 54.0

\section{Masaharu Akao, MD}

(National Hospital Organization Kyoto Medical

Center, Kyoto

Number of reviews: 16

RPS: 50.6

\section{Yoshihiro Seo, MD}

(University of Tsukuba, Ibaraki)

Number of reviews: 13

RPS: 75.0

Previous awards: 2012, 2015, 2016, 2017

\section{Takuya Kishi, MD}

(Kyushu University Center for Disruptive

Cardiovascular Medicine, Fukuoka)

Number of reviews: 11

RPS: 62.0

\section{Shiro Nakahara, MD}

(Dokkyo Medical University Saitama Medical Center, Saitama)

Number of reviews: 14

RPS: 58.5

\section{Junya Ako, MD}

(Kitasato University, Kanagawa)

Number of reviews: 11

RPS: 58.2

Previous awards: 2014, 2015, 2016, 2017

\section{Takashi Kunihara, MD}

(The Jikei University School of Medicine, Tokyo)

Number of reviews: 9

RPS: 53.4

\section{Norihide Fukushima, MD}

(National Cerebral and Cardiovascular Center,

Osaka)

Number of reviews: 9

RPS: 50.0

*The Reviewer Performance Score (RPS) is determined as follows for reviewers who made at least 6 reviews in 2018 (review time limit, 14 days):

*RPS $=[($ Number of reviews completed within 1 week $) \times 2+($ number of reviews completed within 2 weeks $) \times 1+($ number of reviews completed within 3 weeks $) \times 0.2] \times$ quality of review (average) $-($ number of declined reviews $)+($ number of editorial comments published in the Journal $) \times 10$.

The review quality of reviewers was evaluated for each manuscript by an Associate Editor who was in charge of the manuscript (range: 1 3) and the average score used.

The above Reviewers will be awarded by the Japanese Circulation Society with a Certificate of Achievement and an honorarium.

In the year ahead in 2019, we expect a steady increase in the number of submissions, as well as an increase in the scientific impact of the Journal. 